\title{
Light Through the Fog: Using Precision fMRI Data to Disentangle the Neural Substrates of Cognitive Control
}

Authors: Derek M. Smith ${ }^{1}$, Diana C. Perez ${ }^{1}$, Alexis Porter ${ }^{1}$, Ally Dworetsky ${ }^{1}$, Caterina Gratton ${ }^{1,2}$ Affiliations:

Department of Psychology ${ }^{1}$, Northwestern University, Evanston; Illinois

Department of Neurology ${ }^{2}$, Northwestern University, Evanston, Illinois

Acknowledgments: This work was supported by NIH grants R01MH118370 (CG), T32NS047987 (DS), and T32MH067564 (AP).

Correspondence: Caterina Gratton (cgratton@northwestern.edu) 


\begin{abstract}
:
Cognitive control, the ability to engage in goal-related behavior, is linked to frontal, parietal, and cingulate brain regions. However, the underlying function(s) of these regions is still in question, with ongoing discussions about their specificity and/or multifunctionality. These brain regions are also among the most variable across individuals, which may confound multi-functionality with inter-individual heterogeneity. Precision fMRI—extended data acquisition from single individuals-allows for reliable individualized mapping of brain organization. We review examples of recent studies that use precision fMRI to surmount inter-individual variability in functional neuroanatomy. These studies provide evidence of interleaved specialized and multifunctional regions in the frontal cortex. We discuss the potential for these techniques to address outstanding controversies on the neural underpinnings of cognitive control.
\end{abstract}

Highlights

1. Cognitive control areas show high cross-subject variation in functional organization

2. Cross-subject variability can confound reports of control area multifunctionality

3. Precision $\mathrm{fMRI}$ can identify individualized functional regions linked to control

4. This approach may help disambiguate functional accounts of regions such as the dACC

5. We review the use of individualized methods to elucidate the neurobiology of control 
Cognitive control is the set of processes responsible for guiding complex behavior in the pursuit of a goal, allowing us to behave in an adaptive manner. These processes are relevant to important life functions, including emotion regulation, action-planning, and decision-making [1]. Neuroimaging, animal physiology, and human lesion research have made tremendous strides in the search for the neural substrates of cognitive control, linking prefrontal cortex, anterior cingulate cortex, the anterior insula, and parietal regions to control-related functions [2-8]. Interestingly, the brain regions linked to cognitive control exhibit correlated activity patterns, even at rest, which can be detected with functional connectivity MRI (fcMRI, see Box 1; [9-11]) However, the umbrella term "cognitive control" covers a variety of processes ranging from maintenance of a goal representation to inhibition of task-irrelevant inputs. Thus, a deep understanding of cognitive control requires identifying its precise neural substrates, decomposing this complex domain into distinct neural processes and their interrelationships.

A major obstacle to understanding the neural correlates of cognitive control is variability in functional brain organization across individuals. Most fMRI studies of cognitive control assume functional correspondence between anatomical locations across subjects (e.g., creating group average maps based on anatomical alignment), but recent research has demonstrated that individuals can differ substantially in their functional architecture [12,13], particularly in frontal and parietal cortex $[14,15]$. In this review, we will first discuss the fundamental issue that cross-subject heterogeneity poses to the study of cognitive control. We will then present a potential solution: use of a "precision" approach based on the collection of extended datasets $[12-14,16,17]$ to reliably identify functionally-matched brain regions within individual subjects. We review recent studies that have used this approach to help address ongoing debates on specificity and generality of function in frontal lobe brain regions, and suggest avenues for fruitful future applications of this work to improving our understanding of cognitive control.

\section{Box 1}

\section{Functional Connectivity MRI}

Functional connectivity MRI (fcMRI) is a technique that measures temporal correlations in the blood oxygen level dependent (BOLD) signal between different brain regions.

Functionally-related regions not only co-activate during a relevant task, but also exhibit synchronous spontaneous fluctuations during rest. Thus, resting-state fcMRI can be used to identify separate systems based on correlations in their patterns of activity [18].

Biswal and colleagues [18] first observed that the spontaneous activity in primary motor cortex was highly correlated with the homotopic primary motor cortex as well as other cortical and subcortical motor system regions. Since this seminal study, many other canonical systems have been identified [10,11], including ones putatively specialized in cognitive control (the frontoparietal and cinguloopercular control networks $[9,19]$ ) and attention (the dorsal and ventral attention networks [3]). These approaches have also been extended to mapping not only networks, but single brain regions $[16,20]$ based on abrupt changes in functional connectivity patterns between spatially neighboring locations.

FcMRI is advantageous relative to traditional task $\mathrm{fMRI}$ activation studies, as it allows the measurement of many networks at once, without necessitating a separate task to identify each seoarate network. Despite their unconstrained nature, the patterns of spontaneous activity appear to be functionally relevant as they align closely with task-evoked BOLD responses $[13,16]$. Moreover, network maps derived from rest data across multiple independent datasets have been fairly consistent, suggesting these patterns of fcMRI are robust and reflect a common, stable functional architecture $[10,11]$. These findings indicate that fcMRI is a powerful 
method that can provide detailed descriptions of the network topology in the brain that is both stable and relevant to function.

\section{The fog of heterogeneity: how variability in brain organization degrades our} understanding of cognitive control

It is well established that there are inter-individual differences in the anatomical organization of brain regions, activation maps, and network profiles [13-15,21-24]. Recently, a number of studies have used fcMRI to compare patterns of variability in systems-level brain organization across people $[13-15,21,22,25]$. These studies have consistently demonstrated that lateral frontal and parietal regions exhibit the highest degree of inter-individual variability in the cortex $[14,15]$. Importantly, these regions overlap with the locations most consistently implicated in cognitive control $[4,8,19]$ (Figure 1). This leads to an important limitation in group studies of cognitive control: regions that vary across people will be averaged together in the group, leading to the proverbial mixing of "apples and oranges".
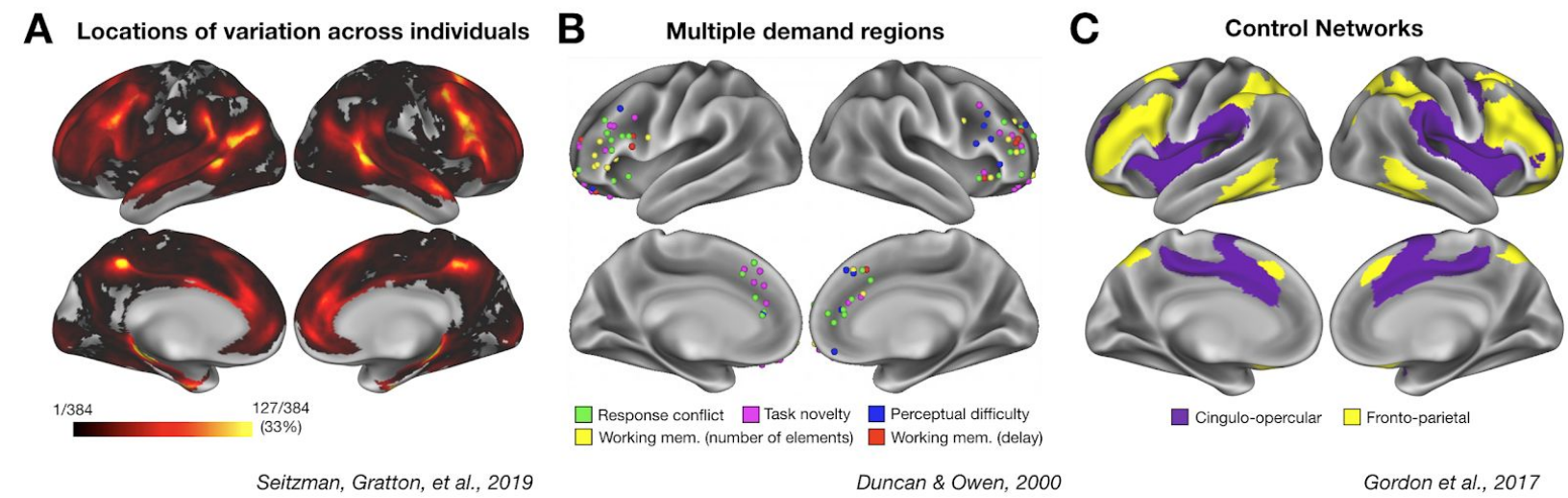

Figure 1: Locations of variation across individuals overlap with locations associated with cognitive control. (A) Locations of the cortex where individuals differ strongly from the typical group architecture (adapted from Seitzman and colleagues [14]; warmer colors represent more common sites of variation, with regions in yellow representing sites that vary in at least $33 \%$ of individuals. (B) Coordinates reported by Duncan and Owen [7] for sites with cognitive control related task activations. (C) The group-level cinguloopercular (purple) and frontoparietal (yellow) "control" networks originally identified by Gordon and colleagues from resting-state fMRI data [13]. Note the overlap between cognitive control regions/networks and locations of individual variability.

Compounding this issue, many of the questions regarding the neurobiology of cognitive control regard the degree of functional specialization of control regions. For example, one long standing argument in the cognitive control literature concerns the dorsal anterior cingulate cortex (dACC), which has been ascribed many functions including error detection [26], conflict monitoring [2,27], task set maintenance [19], time on task [28], tracking the value of behavioral changes [29], updating internal models of the environment [29], and pain perception [30]. There are a number of explanations for the scope of functions associated with the dACC (and extended areas of the medial frontal cortex, $\mathrm{mFC}$ ): 1) The dACC/mFC may be responsible for many separate cognitive functions; 2 ) there may be an underlying common function/process to the diverse set of tasks that have been associated with $\mathrm{dACC} / \mathrm{mFC}$ activation in which these regions are involved; or 3 ) the $\mathrm{dACC} / \mathrm{mFC}$ may be composed of multiple sub-regions with diverse functions that are mixed together across subjects and studies due to heterogeneity in brain organization. FcMRI shows that many distinct networks can be identified in this sub-region (Figure 2), suggesting that there may be multiple sub-components to the dACC/mFC. This heterogeneity is increased when looking at individual data, where a given anatomical location 
may be in different networks across people (Figure 3). Studies examining functional activations in the $\mathrm{dACC} / \mathrm{mFC}$ using group-average maps will then necessarily mix task signals from different sub-regions. This heterogeneity may underlie some of the diverse accounts of multifunctionality and specificity linked to the dACC/mFC (Figure 2).
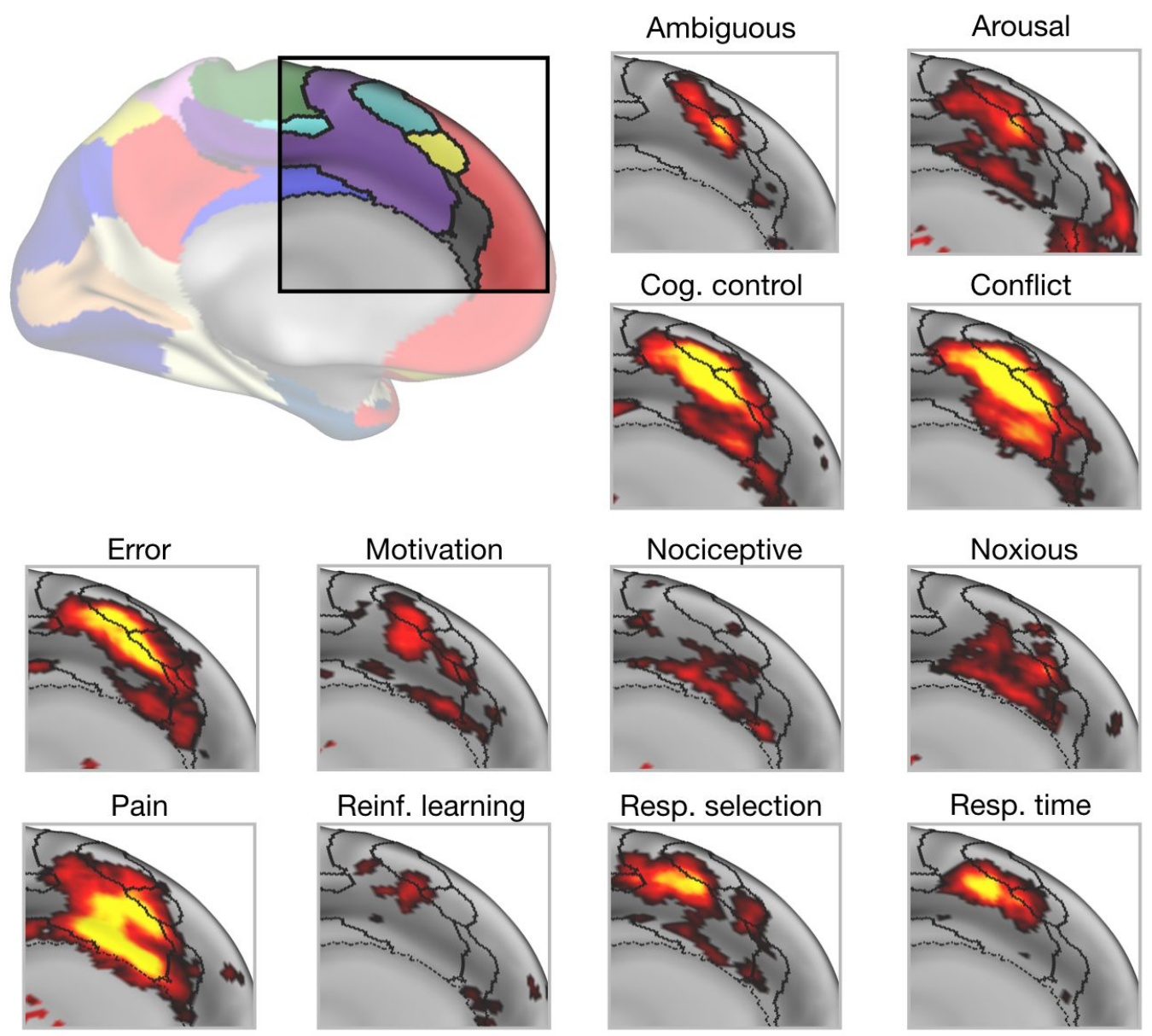

$\mathbf{z}=0$

$$
z=10
$$

Figure 2: Diversity of functional activations in the $d A C C / m F C$. The top left inset shows the functional networks typically identified in the $\mathrm{dACC} / \mathrm{mFC}$ based on group-average data. Other subplots show Neurosynth [31] uniformity images, focused on terms that have been associated with $\mathrm{dACC} / \mathrm{mFC}$ functions in past literature (black lines = borders of functional networks from inset as identified by Gordon and colleagues [13]). Note that many activations overlap with multiple networks and cross network boundaries. Moreover, note also that these group-average networks show substantial variability across individual subjects in Figure 3.

Another major debate concerns whether lateral frontal cortex contains multiple sub-systems specialized for different processes [24,32], or if single regions contribute to generalized aspects of cognitive control (e.g., constituting a "multiple demand" system [7]). Support in favor of a single generalized system for cognitive control came from an early meta-analysis, which showed common activations across many cognitive control tasks [7] (Figure 1). However, counter arguments have suggested the presence of at least two distinct 
sub-systems for cognitive control based on evidence that fcMRI correlation patterns separate into at least two separate control networks (cinguloopercular and frontoparietal $[9,19]$ ), that also exhibit some differences in their task activations, linking them to sustained task set maintenance and adaptive control, respectively (reviewed in [33] Figure 1). However, a detailed understanding of functional differentiation among these networks requires overcoming variability in control network locations across subjects (e.g., Figure 3, purple and yellow).

In both cases, theoretical arguments rely on determining the extent to which a single brain region is specialized or exhibits multi-functionality. However, individual variability in brain organization means that simply aggregating across subjects based on spatial location can lead to averaging signals from different functional regions across subjects, producing a distorted picture of the neural substrates of cognitive control.

\section{Light Through the Fog: using precision fMRI to address issues of heterogeneity}

A strong understanding of the neural underpinnings of cognitive control requires individualized approaches that can identify the same functional sub-region across subjects. "Precision" (or "deep") fMRI methods, which focus on collecting high-quality measurements in single participants through extended data collection and phenotyping, are ideally suited to this endeavor.

One version of this approach is to use task "localizer" scans to individually map brain regions of interest based on their activations in response to a task. There is a long history of vision scientists employing independent localizer scans to identify a visual region based on its functional properties (e.g., for retinotopy or category selectivity [34,35]) separately within each participant. This method allows researchers to bypass cross-subject heterogeneity in anatomical location and focus on functionally-consistent regions across people, and then interrogate the properties of those regions as stimuli or task demands change. An individualized task-localizer approach has also been used to identify higher-level regions with sensitivity to more complex stimuli, for example to identify language-related areas in the frontal cortex [17]. These studies highlight the advantages of accounting for individual variation in anatomical location when investigating functional specificity of brain regions. However, one concern with using this approach in cognitive control research is that, given the diversity and flexibility of control processes, there is little consensus on what task should be used as a localizer to identify control regions (potentially requiring a large number of very diverse tasks for complete mapping).

A different approach is to use fcMRI to identify separate regions or systems based on correlations in their spontaneous activity (Box 1), including those putatively specialized in cognitive control (e.g., the frontoparietal and cinguloopercular networks $[9,19])$ Relative to task localizers, these approaches are appealing as many different networks can be simultaneously extracted from spontaneous resting-state activity, rather than requiring diverse specialized task localizers. While most fcMRI studies are based on group-average data, recently researchers have extended these techniques to define networks and regions in individual subjects through collection of extensive precision-fMRI data sets [12,13,16,36-38]. With a large amount of data, functional connectivity measures can achieve high within-subject reliability (test-retest $r>0.85$ with over $30 \mathrm{~min}$. of low motion data in the cortex [13]), producing detailed individualized maps of brain systems $[12,13,16,38-40]$. Regions identified with precision fMRI approaches have been shown to match individualized visual [16] and motor areas [13] identified based on more traditional task localizer approaches. Importantly, individualized resting-state maps do better at matching motor and visual task activations than do the group-level networks [13]. Thus, these findings highlight the potential that fcMRI has to map individually-specific functional regions without the experimental constraints of a task.

Precision fcMRI has also been used to map higher-level association regions. For example, Braga and colleagues used precision fcMRI to identify and separate closely juxtaposed networks associated with "default mode" locations of the brain, which mapped onto 
differences in task activations associated with episodic projection and social cognition, respectively [39]. This work was recently extended to map a third network associated with language [40]. These studies suggest that precision fcMRI may fruitfully be applied to the study of higher-level functions related to cognitive control due to its ability to produce reliable individualized network measures that map onto individualized differences in task function.

\section{Light through the fog: The potential of precision fMRI in cognitive control}

Using task localizers or fcMRI measures, reliable individual-specific functional brain regions can be defined, combating the issue of cross-subject variability in control-related brain regions. A handful of recent studies exemplify this potential. Assem and colleagues recently asked whether the apparent domain generality of the multiple demand system could stem from heterogeneity in the boundaries of functionally-specific regions across subjects [41]. They created individual-specific regions (defined partly based on fcMRI) to demonstrate that a core set of regions in lateral frontal and parietal cortex exhibited common activations across a variety of diverse tasks in the Human Connectome Project, suggestive of domain generality. However, the individualized data also demonstrated that these locations were more spatially constrained than previously indicated, suggesting that the original approach had been affected by cross-subject heterogeneity, and refining the view of a multiple demand system.

Others have also used individualized fMRI approaches to provide examples of functional specificity within the lateral prefrontal cortex. Fedorenko and colleagues used language task localizers to provide evidence that language-selective regions lie separated but closely adjacent to domain-general regions of lateral frontal cortex [42] - regions that were likely mixed together in previous group studies due to heterogeneity across subjects. Similarly, individualized mapping has uncovered visually-selective and auditory-selective attention regions in the frontal lobe [24], in addition to multiple demand regions [32]. These findings suggest that multifunctional cognitive control regions may lie interspersed with regions that have specific modality or domain specialization. Importantly, the interspersed nature of these regions, and their variability across subjects, suggests that individualized approaches are necessary to tease these regions apart.

The benefits of individualized brain maps are not restricted to the study of distributed cortical territories, but can also help illuminate the function of specific sub-regions in cognitive control. For example, Suda and colleagues individually defined six sub-regions within the right inferior frontal cortex (rIFC) and demonstrated that these sub-regions differ in their involvement in response inhibition [43]. As with the previously described sensory and motor functional sub-divisions, the relationships between the six rIFC sub-regions and cognitive inhibition were generally stronger for individual vs. group-level parcels. Thus, individualized measures can add important information to our understanding of cognitive control at a finer, sub-regional resolution.

These recent studies all demonstrate the power of an individualized approach to improve our understanding of the specialization or multifunctionality of regions involved in cognitive control. A similar approach might be taken to help resolve debates on the function(s) of the $\mathrm{dACC} / \mathrm{mFC}$. Network maps suggest that this part of cortex is home to multiple closely abutted networks, which vary in position across subjects (Figure 3). Reliable individualized mapping, enabled by precision fMRI, can help determine if the wide array of functions mapped to the $\mathrm{dACC} / \mathrm{mFC}$ are reflective of closely interposed but distinct sub-regions or if there are single $\mathrm{dACC} / \mathrm{mFC}$ locations that show multifunctionality. To address these questions, information from individual-level network maps could be combined with task data to probe the differential role of $\mathrm{dACC} / \mathrm{mFC}$ sub-regions in error monitoring, response conflict, and task set maintenance, among many other proposed functions [2,19,28,44].

Similarly, questions regarding the putative task set and adaptive control specialization of the cinguloopercular and frontoparietal networks $[13,23]$ could be clarified by using a precision 
approach to map these networks in single participants. This would overcome heterogeneity driven by differences in anatomical localization of these systems across participants (Figure 3, purple and yellow) and may help to reveal functional specialization, much as Fedorenko and Michalka have done for language and sensory related processes in the frontal cortex [21,24]. By combining precision fcMRI mapping with richly-phenotyped task measures, we will be able to refine our models of task control systems of the brain.

MSC group average

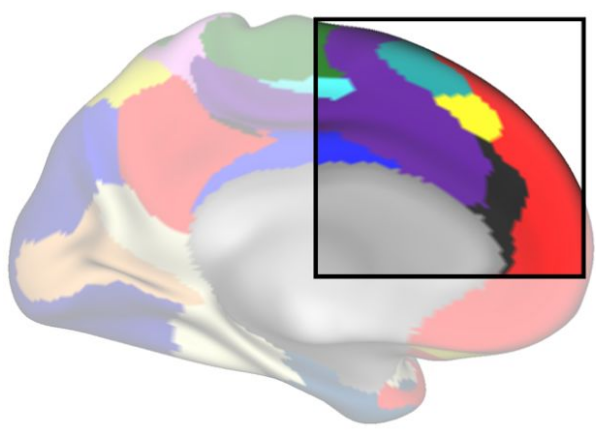

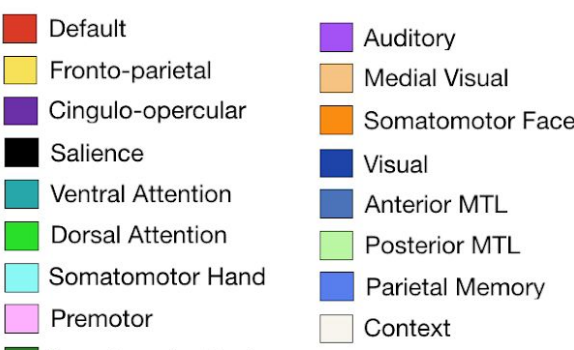

Somatomotor Foot
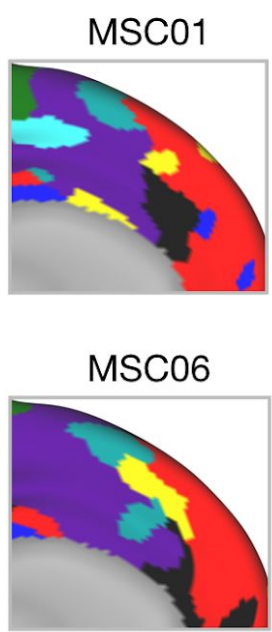
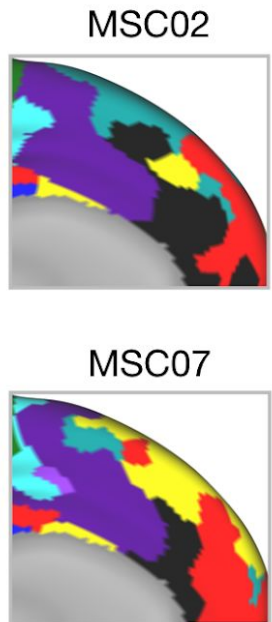
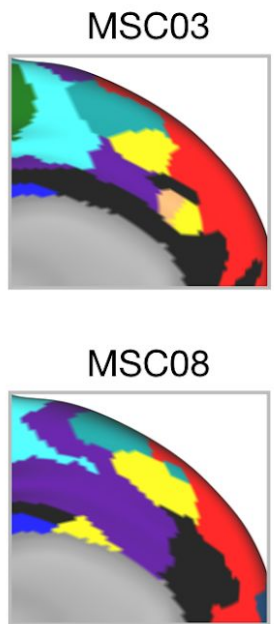
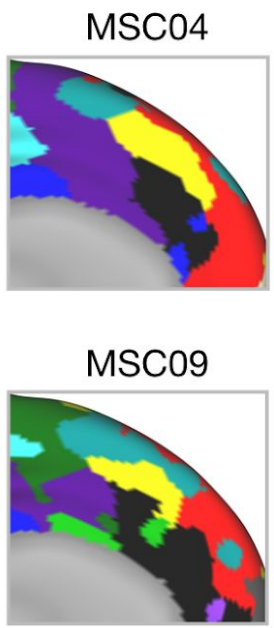
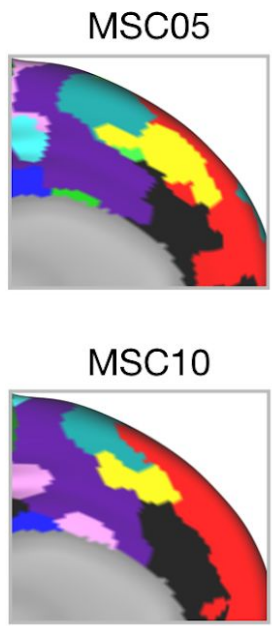

Figure 3: Inter-individual variability in $\mathrm{dACC} / \mathrm{mFC}$ networks. Functional networks (different colors) in the $d A C C / m F C$ on average across a group of individuals in the Midnight Scan Club (top, MSC) or for each individual separately (bottom), derived from resting-state fMRI data [13]. Note the heterogeneity in network assignments across subjects, such that a given position in the group average can be associated with alternate networks in particular subjects.

As these examples demonstrate, the study of the neural bases cognitive control is likely to benefit substantially from individualized precision fMRI. While much of our discussion here has centered on the potential of these techniques to resolve questions of anatomical heterogeneity, these techniques are also well-positioned to enhance our understanding of within and between-person variation in cognitive control abilities (Box 2).

\section{Box 2}




\section{Future Directions: Shedding Light on Within and Between Participant Variability in Cognitive Control}

When regions are defined at an individual level, precision fMRI approaches - which are fundamentally based on extended individual measurements - are also easily amenable to address questions of how these regions respond across different contexts and over time [23]. The transition from controlled to automatic processing is a prime example of a domain of intra-individual variation in control that would benefit from precision fMRI. Most fMRI studies of practice have relied on unpracticed vs. practiced comparison [45-47]. With a large number of within-subject measurements, different time courses of transition from controlled to automatic processing can be observed. Past observations suggest that practice leads to decreased activation in the dACC [45]; a precision $\mathrm{MRRI}$ approach can help investigators determine the temporal nature of reduced $\mathrm{dACC}$ activation and if previously observed practice effects are driven by dACC sub-regions.

Addressing heterogeneity in individual anatomy can also add more power to our understanding of cross-subject variations in cognitive control. There are significant differences in cognitive control performance across individuals $[4,48]$. The improved reliability and specificity of individualized maps enabled by precision fMRI will provide more power to search for brain-behavior relationships that have been obscured in the past by inter-individual variability (Figure 3). This approach can also provide researchers the ability to refine mechanisms that may contribute to brain-behavior relationships, for example probing more deeply into the types of functional or topographic differences in brain organization that may contribute to differences in performance. In this vein, individual-specific brain network topography has been demonstrated to predict cognitive performance [36]. The frontoparietal control network has been suggested to be particularly good at both differentiating individual subjects and predicting fluid intelligence [22]. A recent study by Cui and colleagues used high-data individualized mapping to demonstrate that subtle differences in brain topography in association cortex is predictive of differences in executive function in youth [49]. Although the study of inter-individual variability requires larger datasets than most past precision $\mathrm{fMRI}$ studies [38,50], ongoing and future studies (such as the DMCC Project [51]) that collect precision fMRI data in larger samples will be ideally positioned to help fulfill these needs. This work has substantial promise for improving our understanding of the neural bases of within- and between-subject variations in cognitive control performance.

\section{Summary}

An improved understanding of cognitive control rests on discerning its neural underpinnings, which requires accounting for variability in functional neuroanatomy across subjects. Precision datasets allow researchers to employ robust individualized mapping techniques to shed light on neural heterogeneity across subjects. This added precision makes it possible to weigh multifunctionality accounts of cognitive control regions against specialized function accounts, thereby reducing the uncertainty regarding the role of control-related regions like the $\mathrm{dACC} / \mathrm{mFC}$. Preliminary individualized work has already begun to identify functionally distinct but spatially intertwined sub-systems with different degrees of generality. The enhanced precision of individual-level data is a necessary step to better understanding the functional-neuroanatomical bases of cognitive control. 


\section{Annotated References}

13. * Gordon EM, Laumann TO, Gilmore AW, Newbold DJ, Greene DJ, Berg JJ, Ortega M, Hoyt-Drazen C, Gratton C, Sun H, et al.: Precision Functional Mapping of Individual Human Brains. Neuron 2017, 95:791-807.e7.

The authors used a precision fMRI dataset to evaluate individual specific brain organization and observed that with large amounts of data, individual brain networks can be reliably estimated. Individual differences in rs-FC mapped onto individual differences in task activations for motor and memory-related systems.

14. ${ }^{* *}$ Seitzman BA, Gratton C, Laumann TO, Gordon EM, Adeyemo B, Dworetsky A, Kraus BT, Gilmore AW, Berg JJ, Ortega M, et al.: Trait-like variants in human functional brain networks. Proc Natl Acad Sci USA 2019, 116:22851-22861.

Seitzman et al used a precision rs-FC data to identify locations of individual differences in brain organization relative to a group average. They found that areas of idiosyncratic functional connectivity appear in regions linked to cognitive control.

32. $\quad *$ *Noyce AL, Cestero N, Michalka SW, Shinn-Cunningham BG, Somers DC: Sensory-Biased and Multiple-Demand Processing in Human Lateral Frontal Cortex. J Neurosci 2017, 37:8755-8766.

Noyce and colleagues used an individualized fMRI approach to demonstrate that the frontal cortex contains sensory-biased regions that are interdigitated with multiple demand regions. This work provides evidence for specialized sub-systems within frontal cortex.

41. $\quad{ }^{* *}$ Assem M, Glasser MF, Van Essen DC, Duncan J: A Domain-General Cognitive Core Defined in Multimodally Parcellated Human Cortex. Cerebral Cortex 2020, 30:4361-4380.

Assem and colleagues used an individualized fMRI approach based on the Human Connectome Project dataset to examine whether multiple demand regions were present after accounting for cross-subject differences in brain organization. The authors demonstrate that a distributed set of multiple demands are present, but more restricted than what has been previously reported.

43. * Suda A, Osada T, Ogawa A, Tanaka M, Kamagata K, Aoki S, Hattori N, Konishi S: Functional Organization for Response Inhibition in the Right Inferior Frontal Cortex of Individual Human Brains. Cerebral Cortex 2020, doi:10.1093/cercor/bhaa188.

Suda and colleagues used an individualized fMRI approach to identify sub-regions in the right IFC and examine their responses during a stop-signal task. The authors observed differences in individualized areal organization and sub-regional activity related to response inhibition. 
49. *Cui Z, Li H, Xia CH, Larsen B, Adebimpe A, Baum GL, Cieslak M, Gur RE, Gur RC, Moore TM, et al.: Individual Variation in Functional Topography of Association Networks in Youth. Neuron 2020, 106:340-353.e8.

Cui et al used an individualized approach to examine variability in network topography from childhood to adolescence. The authors found that association network topography was predictive of executive function. 


\section{References}

1. Heitz RP, Unsworth N, Engle RW: Working Memory Capacity, Attention Control, and Fluid Intelligence. In Handbook of Understanding and Measuring Intelligence. . SAGE Publications, Inc.; 2005:61-78.

2. Botvinick MM, Braver TS, Barch DM, Carter CS, Cohen JD: Conflict monitoring and cognitive control. Psychological Review 2001, 108:624-652.

3. Corbetta M, Shulman GL: Control of goal-directed and stimulus-driven attention in the brain. Nat Rev Neurosci 2002, 3:201-215.

4. Kane MJ, Engle RW: The role of prefrontal cortex in working-memory capacity, executive attention, and general fluid intelligence: An individual-differences perspective. Psychonomic Bulletin \& Review 2002, 9:637-671.

5. Levy R, Goldman-Rakic PS: Segregation of working memory functions within the dorsolateral prefrontal cortex. Exp Brain Res 2000, 133:23-32.

6. Müller NG, Knight RT: The functional neuroanatomy of working memory: Contributions of human brain lesion studies. Neuroscience 2006, 139:51-58.

7. Duncan J, Owen AM: Common regions of the human frontal lobe recruited by diverse cognitive demands. Trends in Neurosciences 2000, 23:475-483.

8. Miller EK, Cohen JD: An Integrative Theory of Prefrontal Cortex Function. Annu Rev Neurosci 2001, 24:167-202.

9. Dosenbach NUF, Fair DA, Miezin FM, Cohen AL, Wenger KK, Dosenbach RAT, Fox MD, Snyder AZ, Vincent JL, Raichle ME, et al.: Distinct brain networks for adaptive and stable task control in humans. Proceedings of the National Academy of Sciences 2007, 104:11073-11078.

10. Power JD, Cohen AL, Nelson SM, Wig GS, Barnes KA, Church JA, Vogel AC, Laumann TO, Miezin FM, Schlaggar BL, et al.: Functional Network Organization of the Human Brain. Neuron 2011, 72:665-678.

11. Thomas Yeo BT, Krienen FM, Sepulcre J, Sabuncu MR, Lashkari D, Hollinshead M, Roffman JL, Smoller JW, Zöllei L, Polimeni JR, et al.: The organization of the human cerebral cortex estimated by intrinsic functional connectivity. Journal of Neurophysiology 2011, 106:1125-1165.

12. Braga RM, Buckner RL: Parallel Interdigitated Distributed Networks within the Individual Estimated by Intrinsic Functional Connectivity. Neuron 2017, 95:457-471.e5.

13. Gordon EM, Laumann TO, Gilmore AW, Newbold DJ, Greene DJ, Berg JJ, Ortega M, Hoyt-Drazen C, Gratton C, Sun H, et al.: Precision Functional Mapping of Individual Human Brains. Neuron 2017, 95:791-807.e7. 
14. Seitzman BA, Gratton C, Laumann TO, Gordon EM, Adeyemo B, Dworetsky A, Kraus BT, Gilmore $\mathrm{AW}$, Berg JJ, Ortega $\mathrm{M}$, et al.: Trait-like variants in human functional brain networks. Proc NatI Acad Sci USA 2019, 116:22851-22861.

15. Mueller S, Wang D, Fox MD, Yeo BTT, Sepulcre J, Sabuncu MR, Shafee R, Lu J, Liu H: Individual Variability in Functional Connectivity Architecture of the Human Brain. Neuron 2013, 77:586-595.

16. Laumann TO, Gordon EM, Adeyemo B, Snyder AZ, Joo SJ, Chen M-Y, Gilmore AW, McDermott KB, Nelson SM, Dosenbach NUF, et al.: Functional System and Areal Organization of a Highly Sampled Individual Human Brain. Neuron 2015, 87:657-670.

17. Fedorenko $\mathrm{E}$, Behr $\mathrm{MK}$, Kanwisher $\mathrm{N}$ : Functional specificity for high-level linguistic processing in the human brain. Proceedings of the National Academy of Sciences 2011, 108:16428-16433.

18. Biswal B, Zerrin Yetkin F, Haughton VM, Hyde JS: Functional connectivity in the motor cortex of resting human brain using echo-planar mri. Magn Reson Med 1995, 34:537-541.

19. Dosenbach NUF, Visscher KM, Palmer ED, Miezin FM, Wenger KK, Kang HC, Burgund ED, Grimes AL, Schlaggar BL, Petersen SE: A Core System for the Implementation of Task Sets. Neuron 2006, 50:799-812.

20. Wig GS, Laumann TO, Petersen SE: An approach for parcellating human cortical areas using resting-state correlations. Neurolmage 2014, 93:276-291.

21. Fedorenko E, Duncan J, Kanwisher N: Language-Selective and Domain-General Regions Lie Side by Side within Broca's Area. Current Biology 2012, 22:2059-2062.

22. Finn ES, Shen X, Scheinost D, Rosenberg MD, Huang J, Chun MM, Papademetris X, Constable RT: Functional connectome fingerprinting: identifying individuals using patterns of brain connectivity. Nat Neurosci 2015, 18:1664-1671.

23. Gratton C, Laumann TO, Nielsen AN, Greene DJ, Gordon EM, Gilmore AW, Nelson SM, Coalson RS, Snyder AZ, Schlaggar BL, et al.: Functional Brain Networks Are Dominated by Stable Group and Individual Factors, Not Cognitive or Daily Variation. Neuron 2018, 98:439-452.e5.

24. Michalka SW, Kong L, Rosen ML, Shinn-Cunningham BG, Somers DC: Short-Term Memory for Space and Time Flexibly Recruit Complementary Sensory-Biased Frontal Lobe Attention Networks. Neuron 2015, 87:882-892.

25. Gordon EM, Laumann TO, Adeyemo B, Petersen SE: Individual Variability of the System-Level Organization of the Human Brain. Cereb Cortex 2015, doi:10.1093/cercor/bhv239.

26. van Veen V, Holroyd CB, Cohen JD, Stenger VA, Carter CS: Errors without conflict: Implications for performance monitoring theories of anterior cingulate cortex. Brain and Cognition 2004, 56:267-276.

27. Kerns JG: Anterior Cingulate Conflict Monitoring and Adjustments in Control. Science 2004, 303:1023-1026. 
28. Grinband J, Savitskaya J, Wager TD, Teichert T, Ferrera VP, Hirsch J: The dorsal medial frontal cortex is sensitive to time on task, not response conflict or error likelihood. Neurolmage 2011, 57:303-311.

29. Kolling N, Behrens T, Wittmann M, Rushworth M: Multiple signals in anterior cingulate cortex. Current Opinion in Neurobiology 2016, 37:36-43.

30. Lieberman MD, Eisenberger $\mathrm{NI}$ : The dorsal anterior cingulate cortex is selective for pain: Results from large-scale reverse inference. Proc Natl Acad Sci USA 2015, 112:15250-15255.

31. Yarkoni T, Poldrack RA, Nichols TE, Van Essen DC, Wager TD: Large-scale automated synthesis of human functional neuroimaging data. Nat Methods 2011, 8:665-670.

32. Noyce AL, Cestero N, Michalka SW, Shinn-Cunningham BG, Somers DC: Sensory-Biased and Multiple-Demand Processing in Human Lateral Frontal Cortex. J Neurosci 2017, 37:8755-8766.

33. Gratton C, Sun H, Petersen SE: Control networks and hubs. Psychophysiol 2018, 55:e13032.

34. Kanwisher N, McDermott J, Chun MM: The Fusiform Face Area: A Module in Human Extrastriate Cortex Specialized for Face Perception. J Neurosci 1997, 17:4302-4311.

35. Engel S: Retinotopic organization in human visual cortex and the spatial precision of functional MRI. Cerebral Cortex 1997, 7:181-192.

36. Kong R, Li J, Orban C, Sabuncu MR, Liu H, Schaefer A, Sun N, Zuo X-N, Holmes AJ, Eickhoff SB, et al.: Spatial Topography of Individual-Specific Cortical Networks Predicts Human Cognition, Personality, and Emotion. Cerebral Cortex 2019, 29:2533-2551.

37. Poldrack RA, Laumann TO, Koyejo O, Gregory B, Hover A, Chen M-Y, Gorgolewski KJ, Luci J, Joo SJ, Boyd RL, et al.: Long-term neural and physiological phenotyping of a single human. Nat Commun 2015, 6:8885.

38. Gratton C, Kraus BT, Greene DJ, Gordon EM, Laumann TO, Nelson SM, Dosenbach NUF, Petersen SE: Defining Individual-Specific Functional Neuroanatomy for Precision Psychiatry. Biological Psychiatry 2020, 88:28-39.

39. Braga RM, DiNicola LM, Becker HC, Buckner RL: Situating the Left-Lateralized Language Network in the Broader Organization of Multiple Specialized Large-Scale Distributed Networks. Journal of Neurophysiology 2020, doi:10.1152/jn.00753.2019.

40. DiNicola LM, Braga RM, Buckner RL: Parallel distributed networks dissociate episodic and social functions within the individual. Journal of Neurophysiology 2020, 123:1144-1179.

41. Assem M, Glasser MF, Van Essen DC, Duncan J: A Domain-General Cognitive Core Defined in Multimodally Parcellated Human Cortex. Cerebral Cortex 2020, 30:4361-4380.

42. Fedorenko E, Duncan J, Kanwisher N: Broad domain generality in focal regions of frontal and parietal cortex. Proceedings of the National Academy of Sciences 2013, 110:16616-16621. 
43. Suda A, Osada T, Ogawa A, Tanaka M, Kamagata K, Aoki S, Hattori N, Konishi S: Functional Organization for Response Inhibition in the Right Inferior Frontal Cortex of Individual Human Brains. Cerebral Cortex 2020, doi:10.1093/cercor/bhaa188.

44. Brown JW: Learned Predictions of Error Likelihood in the Anterior Cingulate Cortex. Science 2005, 307:1118-1121.

45. Chein JM, Schneider W: Neuroimaging studies of practice-related change: $\mathrm{fMRI}$ and meta-analytic evidence of a domain-general control network for learning. Cognitive Brain Research 2005, 25:607-623.

46. Kübler A, Dixon V, Garavan H: Automaticity and Reestablishment of Executive Control-An fMRI Study. Journal of Cognitive Neuroscience 2006, 18:1331-1342.

47. Petersen SE, van Mier $\mathrm{H}$, Fiez JA, Raichle ME: The effects of practice on the functional anatomy of task performance. Proceedings of the National Academy of Sciences 1998, 95:853-860.

48. Friedman NP, Miyake A: Unity and diversity of executive functions: Individual differences as a window on cognitive structure. Cortex 2017, 86:186-204.

49. Cui Z, Li H, Xia CH, Larsen B, Adebimpe A, Baum GL, Cieslak M, Gur RE, Gur RC, Moore TM, et al.: Individual Variation in Functional Topography of Association Networks in Youth. Neuron 2020, 106:340-353.e8.

50. Marek S, Tervo-Clemmens B, Calabro FJ, Montez DF, Kay BP, Hatoum AS, Donohue MR, Foran W, Miller RL, Feczko E, et al.: Towards Reproducible Brain-Wide Association Studies. bioRxiv 2020, doi:10.1101/2020.08.21.257758.

51. Braver TS, Kizhner A, Tang R, Freund MC, Etzel JA: The Dual Mechanisms of Cognitive Control (DMCC) Project. Neuroscience; 2020. 